\title{
A need for screening and early diagnosis of breast cancer
}

In women, breast cancer is the most common malignancy diagnosed worldwide [1]. Improved early screening and referral to improved adjuvant therapy can reduce breast cancer mortality rates. Women with a breast mass or abnormal imaging findings should undergo biopsy guided by appropriate imaging [2]. Once the diagnosis is established, it is important to accurately define the initial extent or staging of the disease [3] and the clinical and molecular subtypes of cancer (based on gene expression) since this information will affect treatment recommendations and prognosis $[4,5]$. The staging and molecular subtypes of breast cancer are used to individualize treatment options.

Early screening can be important to identify breast cancers and their subtypes and staging at an early stage of the disease [6]. This can also influence outcomes of treatment and prognosis. Breast cancers detected through screening are diagnosed at an earlier stage and have a greater proportion of subtypes, with better outcome. Variations in survival are found in women with non-screen-detected breast cancer [6]. Ideally, screening that identifies earlier stage of disease can be performed in population setting which can link positive subjects with further diagnosis and treatment in the health care system.

The report by Sripan et al in this volume represents the first population based on clinical subtypes of breast cancers in a northern province in Thailand [7]. The proportion of breast cancer subtypes was different from other populations [7]. Moreover, the proportion of different subtypes changes over time. This can have important bearing on resources to be planned for treatment of breast cancer.

Few patients with metastatic breast cancer are cured. Therefore, screening and early diagnosis is a good strategy to cope with the disease which can occur more commonly as women today have longer life expectancy. Nevertheless, a significant progress has been made in improving the duration of patient survival and their quality of life. Physicians usually resort to a judicious use of "systemic therapy" such as chemotherapy, endocrine therapy, monoclonal antibodies, or some combination of these options. Surgery and radiation therapy can help control disease in certain local metastasis to certain areas, such as metastasis to bone, and brain, and metastasis to other areas causing symptoms and sufferings. These treatments do not cure the disease. It can only serve to prolong life, reduce sufferings, and improve quality of life.

Given the problems associated with delayed diagnosis and treatments which allow the breast cancers to metastasize, the importance of population based screening of breast cancers and their subtypes cannot be overemphasized.

\section{References}

[1] Siegel RL, Miller KD, Jemal A. Cancer statistics, 2019 Cancer J Clin. 2019; 69:7. Epub 2019 Jan 8.

[2] Pisano ED. Digital compared with screen-film mammography: measures of diagnostic accuracy among women screened in the ontario breast screening program-evidence that direct radiography is superior to computed radiography for cancer detection. Radiology. 2016; 278:311.

[3] Parkin DM, Ward K, Chokunonga E, Ervik M, Farrugia H, Gospodarowicz M, et al. Essential TNM: a registry tool to reduce gaps in cancer staging information. Lancet Oncol. 2019; 20:e103-11.

[4] Parise CA, Bauer KR, Brown MM, Caggiano V. Breast cancer subtypes as defined by the estrogen receptor (ER), progesterone receptor (PR), and the human epidermal growth factor receptor 2 (HER2) among women with invasive breast cancer in California, 1999-2004. Breast J. 2009; 15:593-602.

*Correspondence to: Editorial Office of Asian Biomedicine, Faculty of Medicine, Chulalongkorn University, Bangkok 10330, Thailand, e-mail: abmjournal@chula.ac.th

O Open Access. ๑ 2019 Editorial Office of Asian Biomedicine, published by Sciendo. (๔) BY-NC-ND This work is licensed under the Creative Commons Attribution NonCommercial-NoDerivatives 4.0 License. 
[5] Pan XB, Chen RJ, Huang ST, Jiang YM, Zhu XD Systematic review and meta-analysis of the efficacy of breast conservation therapy followed by radiotherapy in four breast cancer subtypes. Oncotarget. 2017; 8:57414-20.

[6] Lawrenson R, Lao C, Jacobson G, Seneviratne S, Scott N, Sarfati D, et al. Outcomes in different ethnic groups of New Zealand patients with screen-detected vs. non-screen-detected breast cancer. J Med Screen. 2019:969141319844801. doi: 10.1177/0969141319844801.

[7] Sripan P, Sriplung H, Pongnikorn D, Bilheem S, Virani S, Waisri $\mathrm{N}$, et al. Clinical subtypes of breast cancer in Thai women: a population-based study of Chiang Mai province. Asian Biomed (Res Rev News). 2019; 13:11-17. 